\title{
FUZZY AUTOMATION CONTROLLER FOR FLEXIBLE MANUFACTURING SYSTEMS
}

\author{
Venkateswaran P.R ${ }^{1}$, Jayadev Bhat ${ }^{2}$, Meenatchisundaram S. ${ }^{3}$ \\ ${ }^{1,2,3}$ Department of Instrumentation and Control Engineering, Manipal Institute of Technology, Manipal, Karnataka \\ Email: prv_i@yahoo.com
}

\section{Abstract}

The behavior mapping of systems has undergone rapid change with advances in technology. The representation has become digital with state description of the behavior. This manifestation is called Discrete Event Systems (DES), exhibiting properties such as non - determinism, conflict and parallelism[1]-[3]. Supervisory Control Theory (SCT)[8],[9] is chosen as a modeling paradigm and Programmable Logic Controllers (PLC) [10] - [12] as an implementation tool for DES. This work investigates the use of Fuzzy Petri nets in supervisory control and suggests a modified and improved version called Fuzzy Automation Petri net (FAPN) as a modeling tool. It presents a systematic approach to the synthesis of Fuzzy Petri net based supervisor for the forbidden state problem using supervisory design procedure. The controlled model of the system can be constructed from this FAPN net structure. The implementation is using Flexible Manufacturing System (FMS) as an example of DES. The results can be interpreted and applied to high level manufacturing systems, where the role of the supervisor is to coordinate the control of multiple machines or to low level manufacturing systems, where the control function is to switch ON/OFF with respect to the dynamics between different valves.

Keywords: FMS, Fuzzy Petri nets, PLCs,

\section{INTRODUCTION}

The Flexible Manufacturing System shown in Figure 1 represents a packaging process that can be controlled by a Programmable Logic Controller. In this application the objective is to control the speed of two conveyors which are used for packaging products. The strategy is to optimize the speed of a motor driven belt conveyor so that productivity in terms of packaging is achieved.

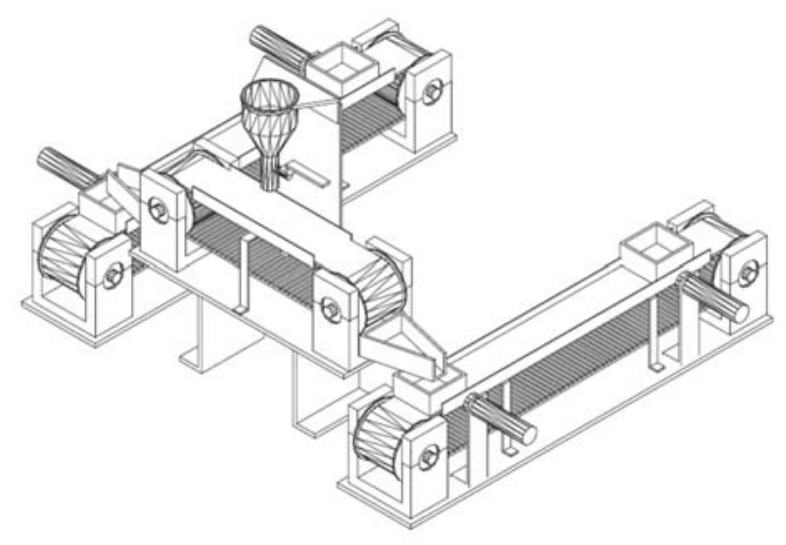

Fig. 1. Schematic of the FMS considered for operation.

The FMS trainer in Figure 1 consists of the following:

- $7 \mathrm{Kg}$ stepper motor of two nos. to drive the conveyor
- Four nos. of timer pulleys

- Optical sensor to sense the wooden object

- Proximity sensor to sense the metal object

- Three nos. of pneumatic operated cylinder for stamping and pushing the object

- One Air regulator, three nos. of single ended solenoid valve and a mini air compressor.

- Air hose and accessories.

- Pulley diameter : $60 \mathrm{~mm}$

- Belt width : 50mm

- Centre distance b/w two pulley : 440mm

Products are carried at irregular intervals in both the right conveyors $\left(C_{R}\right)$ and left conveyor $\left(C_{L}\right)$. The speeds of both the conveyors are controllable. The processes in the conveyors $C_{R}$ and $C_{L}$ are identical and are as follows: Both the conveyors are started together and the system will wait for a signal from either $S_{R}$ or $S_{L}$. Suppose $S_{R}$ (a box is detected on conveyor R) occurs (if not it will check for left conveyor), the Hopper feed conveyor is moved towards right $(H C R)$. Now if the box has arrived at $D_{R}\left(D_{R}\right.$ occurs), the Hopper Valve is opened $\left(V_{1}\right)$. The valve remains open for time $T$ and then it is closed $\left(V_{0}\right)$. The conveyors are again started and the flow starts. In case of the box is detected first at conveyor $L$ ( $S_{L}$ occurs), the same sequence is followed at the left conveyor. 
The most important thing to note here is that the time interval $A_{1}$ (i.e. the interval between the instant $S_{R}$ occurs and $D_{R}$ occurs), a control action needs to be such that both $C_{R}$ and $C_{L}$ are controlled. The same procedure is true for the other cycle. A Siemens PLC (CPU 226) is used to control the process and Step 7 Version 4.0 is used as the software interface for the PLC.

\section{CONTROLLER SYNTHESIS USING THE INHIBITOR ARC METHOD}

Fuzzy Automation Petri net (FAPN) is a variant of APN in [13], [16] and is used to capture the uncontrolled behavior of the system. The representation of the system using FAPN is as in Figure 2.This is derived as a result of study of [4] - [7]. There are fifteen places, $P=\left\{p_{0}, p_{2} \ldots p_{14}\right\}$ and ten transitions, $T=\left\{t_{1}, t_{2} \ldots t_{10}\right\}$, with firing conditions defined as $\chi_{1}=10.0, \quad \chi_{2}=10.0, \quad \chi_{3}=10.1, \quad \chi_{4}=10.2, \quad \chi_{5}=10.3$, $\chi_{6}=10.4, \chi_{7}=10.5, \chi_{8}=10.6$ associated with them respectively for the first eight transitions. The last two of the transitions are defined by timers. The places $p_{0}$ and $p_{2}$ define the on and off status of left conveyor. Similarly, the places $p_{1}$ and $p_{3}$ define the on and off status of right conveyor. A token in the place of $p_{4}$ or $p_{5}$ indicates the presence of box sensed at the entry of either the left or the right conveyor. A token in place of $p_{9}$ or $p_{10}$ indicates the running of the Hopper Feed Conveyor towards the left or right. The Hopper feed valve status is indicated by places $p_{11}$ and $p_{12}$. The following is the recall of the description of the system. The initial conditions for the system are the conveyors $\left(C_{L}\right.$ and $\left.C_{R}\right)$ switched off, the Hopper Feed Conveyor (HFC) is idle, the Hopper Feed Valve (HFV) is closed and the buffer of $C_{L}$ and $C_{R}$ are full. Conveyors $C_{L}$ and $C_{R}$ can be started with a start switch. Whenever a box is detected at the entry of the either of the conveyor, the Hopper Feed conveyor if ready should start moving towards that particular conveyor. If in case the Hopper Feed Conveyor is already engaged, it should wait till the operation gets completed. The box travels to the filling station where it gets filled through the hopper and then finally gets dispatched.

The reachability graph of the uncontrolled FAPN model is shown in Figure 3, where there are 57 arcs representing the firing of transitions in the uncontrolled model and there are 19 nodes $M=\left\{M_{0}, M_{1} \ldots M_{18}\right\}$ representing the all possible markings reachable from the initial marking $M_{0}$. The Table provides detailed information about reachability graph nodes. The events $\chi=\left\{\chi_{1}, \chi_{2} \ldots \chi_{8}\right\} \quad$ represents the firing of corresponding transitions $T=\left\{t_{1}, t_{2} \ldots t_{10}\right\}$ respectively. All time delays associated with the transitions are implied although not indicated. "Bad states" in a reachability graph will happen under two circumstances: (i) states which are not to be reached as per specifications, identified as forbidden state specifications (ii) states which are in conflict with the constraints of the system parameters. Initially, the system specifications are considered for defining the "bad states". Such system specifications are called as forbidden state specifications and are denoted as follows:

(i) The boxes should not leave the conveyor without getting filled.

(ii) The Hopper feed conveyor should not respond to transition without completing the current operation.

(iii) The Hopper Feed conveyor should not run till the conveyor in which the box is detected is stopped.

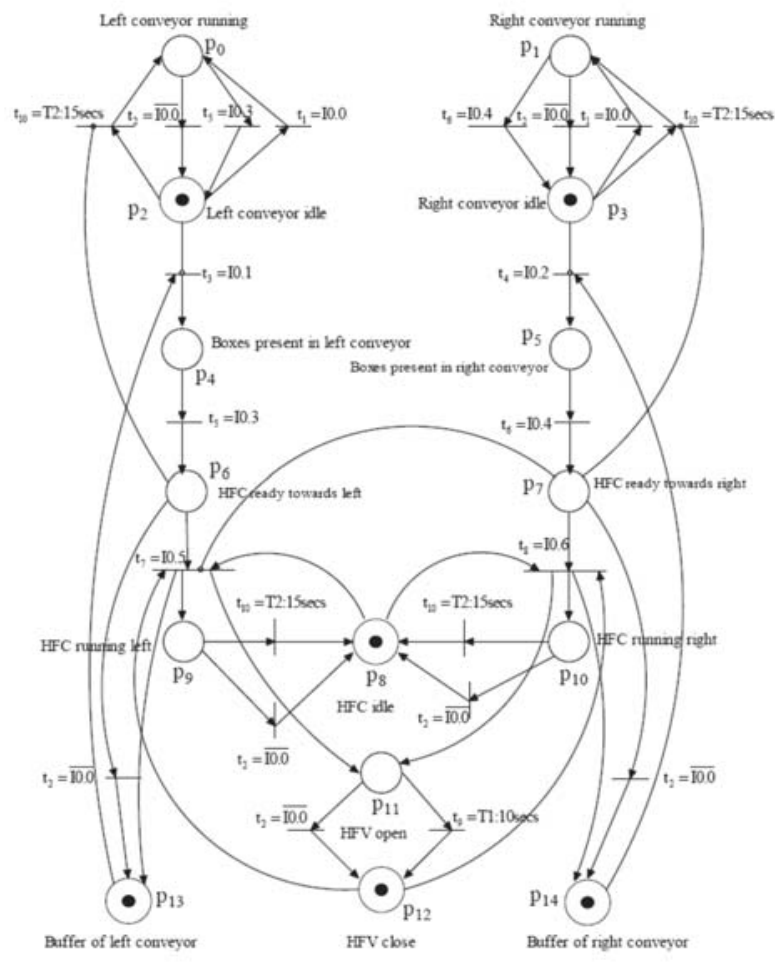

Fig. 2. FAPN model of the Flexible Manufacturing System 


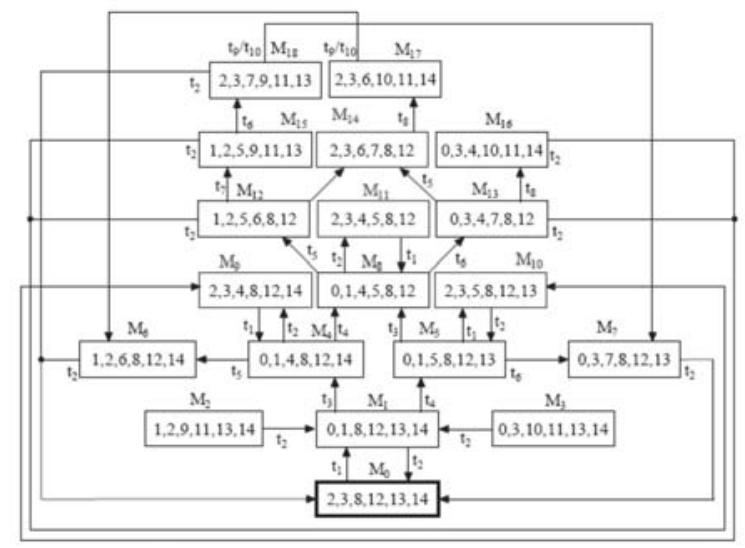

Fig. 3. Reachability graph of the model

To identify the system with respect to its specifications, all of these specifications are considered. In addition, the constraints imposed on the system due to operating/working conditions are also considered. Elimination of states which do not append to any of these specifications is done in the reachability graph. When a state is removed, the transitions that lead/coming from the other states to the specified become redundant and lose their meaning. Hence all the transitions leading to/from "bad states" are also eliminated. For example, let us consider a case of $M_{14}=[2,3,6,7,8,12]$. Here all the conveyors are in idle state. This indicates that all the conveyors are idle simultaneously inferring that no useful work is done in the system. This must be prevented from happening although it is not against specifications per se. Hence, apart from $M_{0}$, the states $M_{9}, M_{10}, M_{11}, M_{14}$ in which all the conveyors become idle are to be removed from the reachability graph. This includes all transitions terminating and originating from the individual states. In addition, there is a state called $M_{16}$ which when reached in the system does not lead anywhere. This is because there is no transition that originates at $M_{16}$. Hence, it should be made sure that $M_{16}$ does not occur. If $M_{16}$ is to be removed, then as a natural extension, $M_{13}$ is also to be removed. Similarly, for states $M_{2}$ and $M_{3}$, there are no input transitions. They cannot happen in the flow of the system. The final graph makes sure that for all defined states, there is a sequence which will be followed to complete the operation. By following the above sequences, the following objectives will be achieved:
1. If there is no sign of machine starving or blocking, then keep the production surplus close to zero. In other words, produce at a rate more or less equal to demand.

2. If an undesirable event (upstream or downstream buffer full or empty) is about to occur, then ignore surplus levels and try to prevent starting or blocking by increasing or decreasing the production rare accordingly.

\section{DESIGN OF THE FAPN MODEL SUPERVISOR AND DETERMINE THE CONTROL POLICY}

The FAPN supervisor model is designed by converting the FRRG into a related FAPN such that every state (or marking) of the FRRG is represented by an FAPN place and the arcs of the FRRG are represented by FAPN transitions. Note that in case of special FAPN, there are no actions assigned to places, because the FAPN model supervisor designed in this way behaves as a monitor that represents the current state of the system. The initial marking is also represented by a token in the FAPN place representing the initial state. When this technique is applied to the manufacturing system, the FRRG is converted into the FAPN model supervisor as shown in Figure 4. The FAPN model supervisor has 10 places $P=\left\{p_{15}, p_{16}, \ldots p_{24}\right\} 15$ transitions roman. The initial marking of the FAPN model supervisor is $M_{0}=(15)$ initially, there is a token in place $p_{15}$. Note that each place within the FAPN model supervisor represents an admissible marking of the FAPN model of the manufacturing problem. The control policy is determined for this manufacturing problem. It represents the actions that the supervisor needs to take on the system when the states change continuously. This may either be enabling or disabling of events because it is possible to reach to the bad markings from good markings. The "bad states" are in fact only removed from the reachability graph but nothing has been done to null the transitions that lead to these bad states. In order to make sure that the correct system operation each event leading from a "good state" to a "bad state" must be stopped. This constitutes the control policy. For example, the bad marking $M_{2}$ is possible to be reached from $M_{1}$ through the controllable event $\chi_{5}$. Therefore the control policy for M1 must be stop $\chi_{5}$ so that the bad marking $M_{2}$ will not be reached. 


\section{CONSTRUCTION OF THE CONTROLLED MODEL OF THE SYSTEM}

The controlled model of the system is obtained by combining the uncontrolled model of the system with the supervisor through the control policy. The controlled model consists of the FAPN model, The FAPN model supervisor and the control policy, which is implemented as inhibitor arcs. The inhibitor arcs are connected from the places of the FAPN model supervisor to the controllable transitions of the FAPN model such that the control policy is satisfied. This is done by connecting the arcs $\ln \left(p_{16}, t_{5}\right), \ln \left(p_{16}, t_{6}\right), \ln \left(p_{18}, t_{2}\right)$, $\ln \left(p_{17}, t_{1}\right), \quad \ln \left(p_{19}, t_{1}\right), \quad \ln \left(p_{20}, t_{1}\right), \quad \ln \left(p_{21}, t_{2}\right), \quad \ln \left(p_{21}, t_{6}\right)$, $\ln \left(p_{22}, t_{1}\right), \quad \ln \left(p_{23}, t_{7}\right), \quad \ln \left(p_{23}, t_{8}\right), \quad \ln \left(p_{24}, t_{7}\right), \quad \ln \left(p_{24}, t_{8}\right)$ where $p_{n}$ indicate places and $t_{m}$ indicate the controllable transitions. This scheme of the supervisor obtained does not contradict the forbidden state specifications and hence the behavior is said to be non blocking. All events that do not contradict the forbidden state specifications are allowed to happen making the supervisor maximally permissive. It can be verified to be correct by construction. The Fuzzy Automation Petri net (FAPN) is converted into Token Passing Logic Controller (TPLC) by assigning flags to places, whose capacity is one, by assigning counters to places, whose token capacity is more than one and by assigning timers to timed transitions. The FAPN in the controlled model do not exhibit timers because the monitoring is done by the supervisor.

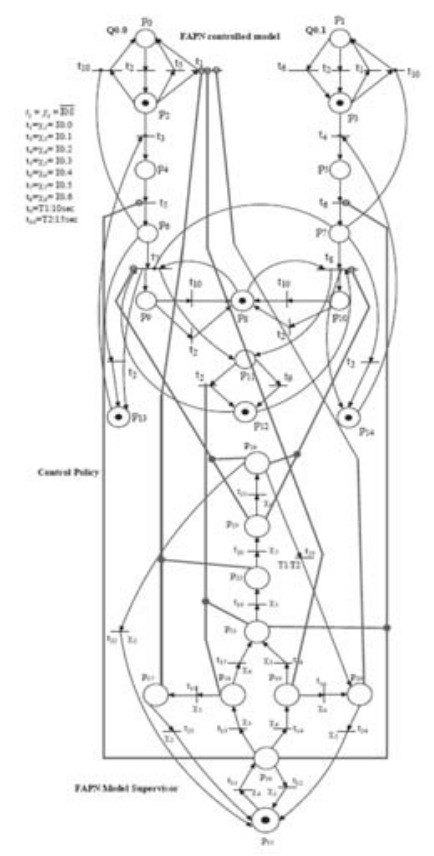

Fig. 4. FAPN model of the system with supervisor
On the other hand, in the FAPN model there are actions assigned to places and on delay timers are also associated with timed-transitions to realize the timing requirements. Next, the TPLC obtained is converted into LLD for implementation on a PLC. To do this, a direct mapping is used from TPLC to LLD code [14]. However, it should be noted that for proper functioning the order of the LLD code, must be arranged as follows: first, the initial marking is written, next, the LLD code related to the FAPN model supervisor is to be written; and finally, the LLD code for the FAPN model is written[15]-[16]. This is because after the initial marking is represented as LLD, the FAPN model supervisor monitors the system behavior and changes its state, and then according to the current state and the control policy, the behavior of the FAPN model is restricted if necessary. Note that, while on delay timers are only associated with the timed transitions in the FAPN model, the time evolution of these timers are followers by the timed - transitions within the FAPN model supervisor. The relative merits of the method could be compared with the peer methods as stated in Table 1.

Table 1. Performance metrics of the methods used

\begin{tabular}{|l|c|c|c|c|}
\hline & $\begin{array}{c}\text { Ladder } \\
\text { Logic } \\
\text { Method }\end{array}$ & $\begin{array}{c}\text { Inhibitor } \\
\text { Arc } \\
\text { method }\end{array}$ & $\begin{array}{c}\text { Enabling } \\
\text { Arc } \\
\text { method }\end{array}$ & $\begin{array}{c}\text { Fuzzy } \\
\text { Automation } \\
\text { Petri net } \\
\text { method }\end{array}$ \\
\hline $\begin{array}{l}\text { Number of } \\
\text { places used }\end{array}$ & NA & 14 & 14 & 12 \\
\hline $\begin{array}{l}\text { Number of } \\
\text { transitions } \\
\text { used }\end{array}$ & NA & 14 & 14 & 10 \\
\hline $\begin{array}{l}\text { Number of } \\
\text { LLD rungs } \\
\text { used }\end{array}$ & 15 & Infinite & Infinite & 140 \\
\hline
\end{tabular}

v. CONCLUSION

A formal design of Fuzzy Petri net based complied supervisors for DES control problem and their efficient implementations are a challenging problem. In this paper, a Flexible Manufacturing System has been considered to illustrate the applicability, strengths and drawbacks of the design techniques proposed. The inhibitor arc method is suggested to solve the design problem. The problem of state space explosion is overcome by using Fuzzy Automation Petri nets. In 
addition, the supervisors obtained are correct by construction. The design was based on the premise that the DES considered are controllable and observable and the result can be extended into a wide array of DES.

\section{REFERENCES}

[1] Anders Hellgren, 2000 Modeling and Implementation aspects of supervisory control, Technical Report No.350L, Control and Automation Laboratory, Department of Signals and Systems, Chalmers University of Technology, Sweden.

[2] Cohen G., Dubois D., Quadrat J.P. and Viot M., 1985, "A Linear System Theoretic View of Discrete Event Processes and its use for performance evaluation in manufacturing", IEEE Trans. on Automatic Control, Vol. AC-30, pp.210-220.

[3] R Valette R., Cardoso J., Dubois D., September 1989 Monitoring Manufacturing Systems by means of Petri nets with imprecise markings, IEEE International Symposium on Intelligent Control, USA, pp. 233 - 238.

[4] Shih Sen Peng, November 2004 Ladder Diagram and Petri - net based Discrete Event Control Design Methods, IEEE Transactions on Systems, Man and Cybernetics - Part C: Applications and Reviews, Vol. 34, No.4.

[5] David R and Alla H. 1992, Petri nets for Modeling of Dynamic Systems: A Survey", Automatica, Vol.30, No.2, pp.175-202.

[6] Tiehua Cao, Arthur C. Sanderson, 1994 Modeling of Sensor Based Robotic Task Plans using Fuzzy Petri nets, Proceedings of IEEE.

[7] Hanna M., 1994 Determination of Product Quality from an FMS Cell using Fuzzy Petri nets, Proceedings of IEEE.

[8] Ramadage P.J. and Wonham W.M., 1987 Supervisory Control of a class of Discrete Event Processes, SIAM Journal of Control and Optimization, Vol.25(1), 206-230.

[9] Ramadage P. J. and Wonham W.M.,1989 The Control of Discrete Event Systems, Proceedings of the IEEE, Vol. 77(1), 81-98,
[10] Taholakian A. and Hales W.M.M. 1997 "PN?PLC: A methodology for designing, simulating and coding PLC based control systems using Petri nets" Intl. J. Product. Res., Vol.35, no.6, pp.1743-1762.

[11] Courvoisier M., Valette R., Bigou J.M. and Esteban P., 1983, "A Programmable Logic Controller Based on a High Level Specification Tool", Proc. of IEEE Conf. on Industrial Electronics, pp. 174-179.

[12] Valette R., 1983, "A Petri Net Based Programmable Logic Controller", In E.A. Warman, (Ed.), Computer Applications in Production and Engineering (CAPE'83), North-Holland Publishing Co., pp. 103-116.

[13] Greene J., 1989-1990, "Petri Net Design Methodology for Sequential Control", Measurement Control, Vol. 22, December/January 22, pp.288-291.

[14] Satoh T., Oshima H., Nose K. and Kumagai S., 1992, "Automatic Generation System of Ladder List Program by Petri Net", Proc. of the IEEE Int. Workshop on Emerging Technologies on Factory Automation Technology For The Intelligent Factory, pp. 128 - 133.

[15] Taholakian W.M. and Hales M., 1995, "The Design and Modeling of PLC Programs Using Petri Nets", Proc. of the Int. Conf. on Planned Maintenance, Reliability and Quality Assurance, Cambridge, England, pp. 194 - 199.

[16] Murat Uzam, 1998 Petri net supervisory control of discrete event systems and their ladder logic diagram implementation, PhD Theses, University of Salford.

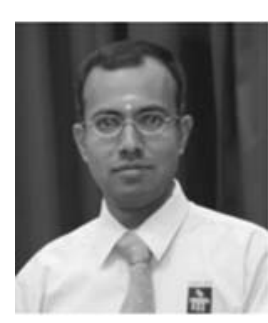

Venkateshwaran is a reader at the department of Instrumentation and Control from Manipal Institute of Technology, Manipal. He is a doctorate from Manipal Institute of Technology, post graduate in Instrumentation and Control from ThaparUnvieristy and a graduate from ManonmaniamSundaranarUniversity. He has over 12 years of teaching experience. He has to his credit 30 publications in International journal, international conferences and national conferences. He is recipient of Research Incentive Award from ManipalUniversity for Journal Publications 